\title{
Two Time Point Analysis of the Change in Risk and Aging Factors for Major Cancers: A 10-Year Longitudinal Study in China
}

\author{
Liu Hui \\ Department of Clinical Immunology, Dalian Medical University, Dalian 116044, China \\ Correspondence should be addressed to Liu Hui; immunology@dmu.edu.cn
}

Received 16 September 2019; Revised 17 April 2020; Accepted 23 April 2020; Published 7 May 2020

Academic Editor: Malay Kumar Basu

Copyright (c) 2020 Liu Hui. This is an open access article distributed under the Creative Commons Attribution License, which permits unrestricted use, distribution, and reproduction in any medium, provided the original work is properly cited.

\begin{abstract}
Objective. To quantify the change in risk and aging factors with a two time point analysis for major cancers to assess supportive strategies. Methods. The 2004 and 2015 mortality statistics in China were accessed. The standardized mortality rates of the two periods were used to calculate the ratio of change (RC) value to assess the risk of death associated with time (social development with time) for cancers. The role of age in mortality with time was evaluated by the interaction between time and age using a Poisson regression. Results. In ascending order of RC, the factors were uterus; other malignant neoplasms; esophagus; stomach; skin; liver; leukemia; "lip, oral cavity, and pharynx"; bladder; "colon and rectum"; breast; prostate; lung; ovary; pancreas; "lymphoid, hematopoietic, and related tissue"; and cervix cancers. According to their location on the scatter diagram, the 17 neoplasms could be divided into three groups, comprising undeveloped cancers (including four cancers), developed cancers (including three cancers), and cancers insensitive to social development. Unexpectedly, about $60 \%$ (as assessed by type of cancer) and two-thirds (as assessed by constituent ratio of death from all cancers) of cancers did not change with time. Conclusions. Most cancers may be insensitive to social development. Internal factors, including aging, may be a key factor for the occurrence of cancer.
\end{abstract}

\section{Introduction}

Cancers are complex disorders, and it is virtually impossible to prove what causes a particular cancer, because most cancers have multiple possible causes. However, we can classify cancer-related factors as risk or protective factors to assess preventative strategies. A hitherto unknown number of risk and protective factors contribute to cancer development by interacting with each other. Although cancer can develop at any age, cancer incidence increases with age [1-3]; therefore, it is believed that old age is the most important risk factor for cancer development and was treated as a major variable in the present study.

Worldwide, the rates of cancer are increasing, mainly related to the aging population and changes in lifestyle resulting from social development [4-6]. Some cancers seem to be more prevalent in people with a lower quality of life, whereas other cancers may be more prevalent in people with a higher quality of life [7]. Social development or urbanization will result in changes to cancer risk and pro- tective factors, including aging, diet and obesity, lack of physical activity, smoking, radiation, infections, stress, and environmental pollutants [8-10], and their impact on the occurrence of cancer [11-13]. Therefore, in growing economies, whether the time trend of increased social development might influence the development and burden of cancer should be explored, which will allow us to identify and implement appropriate medical strategies. To date, there have been no comprehensive and quantitative two time point analyses on cancers.

The cancer incidence $(201.7 / 100000)$ in China is similar to that of the overall worldwide incidence $(197.9 / 100000)$ in 2018 [14]. China's ethnic composition is relatively simple and is presently undergoing rapid urbanization [15-17]. China's gross domestic product was 1.955 trillion USD in 2004 and 11.016 trillion USD in 2015 (Figure 1). Health and medical care has been markedly affected by economic growth, making China an ideal model to study disease patterns associated with modernization [18-20]. In this study, we analyzed the characteristics of cancers using two 


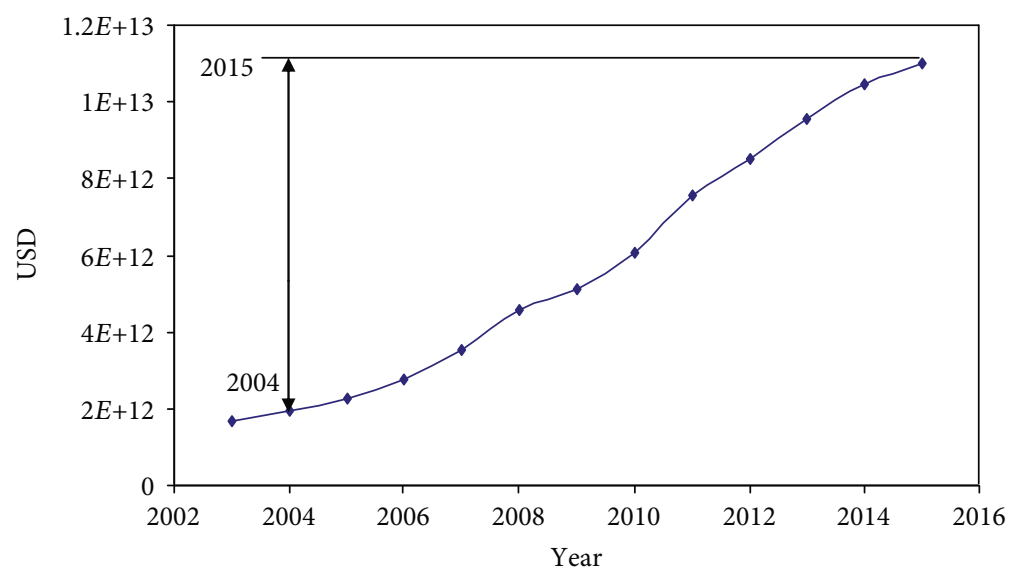

FIgURe 1: Gross domestic product from 2003 to 2015 in China.

properties: (1) age [8] and (2) risk factors [7]. The results showed that although these two properties varied significantly among some cancers, the rates of most cancers were unchanged with time. We also categorized 17 major cancers using two time point analysis and found that about $30 \%$ of cancers were sensitive to social development, and more than $60 \%$ of cancers were insensitive to social development. An improved understanding of such associations would benefit the development of a theoretical basis for cancer prevention and control strategies.

\section{Materials and Methods}

2.1. Original Data. Original data was obtained from the 2004 and 2015 data sets of the national disease mortality surveillance system, which was edited by the Chinese Center for Disease Control and Prevention [21, 22], representing data obtained from over 73 million out of the 1.4 billion population of China between 2004 and 2015. In 2004, life expectancy at birth was 73.6 years, whereas in 2015, it was 76.1 years. The International Classification of Diseases- (ICD-) 10 codes [23] were used to classify the underlying causes of death, allowing us to determine the mortality statistics. Tables 1 and 2 show the raw data.

2.2. Two Time Point Analysis of Deaths Caused by Neoplasms. The mortality rates in 2004 and 2015 were standardized using the 2000 Standard in China [7] and were used to calculate the ratio of change (RC) with time with respect to deaths caused by a particular cancer. The RC values were calculated using the following formula:

$$
R C=\frac{P_{1}}{P_{0}}
$$

where $P_{1}$ represents a cancer's mortality rate in 2015 and $P_{0}$ represents the cancer's mortality rate in 2004.

An $\mathrm{RC}$ value $>1$ indicated that the mortality rate had increased with time, whereas a value $<1$ indicated a decreasing trend with time. To evaluate the degree of deviation from 1 (the values greater than 1 and those less than 1 were com- parable), logarithmic transformation was used to transform the RC values into logarithms, with the absolute value, $\ln R C$, serving as the quantitative value for the two time point analysis.

A scatter diagram was plotted using the $\ln R C$ values. The degree of deviation from 1 and the positions of the various cancers on the scatter diagram were used to sort neoplasms according to the changes in the mortality rate with time.

2.3. Assessing the Role of Age on Mortality from Cancer over Time. To evaluate the mortality risk from aging and social development, a loglinear model using the Poisson regression was employed, with covariates of time (2004 and 2015) and a categorical age of 60 years old [7]. Poisson regression is a generalized linear model form of regression analysis used to model count data and contingency tables. Poisson regression assumes the response variable $Y$ has a Poisson distribution and assumes the logarithm of its expected value can be modeled by a linear combination of unknown parameters. From the model, the $B$ coefficients for the interaction between time and age were obtained, in which $\exp (B)$ was taken as the risk ratio. The greater the positive value of $B$, the greater the effect of the aging factor with time was on a particular cancer [7].

A probability of a type I error $\leq 0.05$ was considered statistically significant. SPSS ver. 17.0 software for Windows (IBM, Armonk, NY, USA) was used to analyze the data statistically.

\section{Results}

The two time point analysis of deaths caused by neoplasms is summarized and quantified in Table 3. The contributions of time (social development) to different cancers varied: The $\ln \mathrm{RC}$ value for the 17 neoplasms ranged from -1.04 to 0.38 . The greater the $\ln \mathrm{RC}$, the greater the disease risk over time. In ascending order of $\operatorname{lnRC}$ were uterus; other malignant neoplasms; esophagus; stomach; skin; liver; leukemia; "lip, oral cavity, and pharynx"; bladder; "colon and rectum"; breast; prostate; lung; ovary; pancreas; "lymphoid, hematopoietic, and related tissue"; and cervix cancers. 
TABle 1: Age-stratified number of deaths from major cancers in 2004 in China [21].

\begin{tabular}{|c|c|c|c|c|c|c|c|c|c|c|c|c|c|c|c|c|c|c|c|}
\hline \multirow{2}{*}{ Age } & \multicolumn{18}{|c|}{ Cancers } & \multirow{2}{*}{ Survival } \\
\hline & A & B & $\mathrm{C}$ & $\mathrm{D}$ & $\mathrm{E}$ & $\mathrm{F}$ & G & $\mathrm{H}$ & I & $\mathrm{J}$ & K & $\mathrm{L}$ & M & $\mathrm{N}$ & $\mathrm{O}$ & $\mathrm{P}$ & Q & $\mathrm{R}$ & \\
\hline $0-$ & 0 & 0 & 0 & 0 & 1 & 0 & 0 & 2 & 0 & 0 & 0 & 0 & 0 & 0 & 2 & 35 & 13 & 53 & 688115 \\
\hline $1-$ & 1 & 0 & 0 & 0 & 7 & 0 & 0 & 2 & 0 & 0 & 0 & 0 & 0 & 1 & 3 & 69 & 6 & 89 & 3030920 \\
\hline 5- & 0 & 0 & 0 & 1 & 7 & 0 & 2 & 2 & 0 & 0 & 0 & 0 & 0 & 0 & 5 & 95 & 5 & 117 & 4604931 \\
\hline $10-$ & 1 & 0 & 0 & 3 & 16 & 0 & 2 & 0 & 0 & 2 & 0 & 2 & 1 & 1 & 9 & 146 & 9 & 192 & 6443682 \\
\hline $15-$ & 10 & 0 & 7 & 12 & 34 & 2 & 22 & 2 & 1 & 1 & 2 & 5 & 1 & 0 & 16 & 220 & 13 & 348 & 5900624 \\
\hline $20-$ & 11 & 8 & 15 & 24 & 64 & 0 & 18 & 2 & 5 & 2 & 5 & 1 & 4 & 1 & 15 & 157 & 14 & 346 & 5467254 \\
\hline $25-$ & 24 & 11 & 53 & 47 & 141 & 6 & 60 & 3 & 20 & 6 & 14 & 9 & 1 & 1 & 19 & 125 & 24 & 564 & 6299615 \\
\hline $30-$ & 69 & 27 & 123 & 83 & 422 & 20 & 165 & 11 & 55 & 32 & 35 & 12 & 2 & 5 & 22 & 163 & 35 & 1281 & 6992522 \\
\hline 35- & 114 & 81 & 302 & 123 & 777 & 41 & 365 & 12 & 136 & 65 & 69 & 13 & 0 & 9 & 54 & 205 & 44 & 2410 & 6360862 \\
\hline $40-$ & 149 & 189 & 436 & 148 & 1144 & 45 & 575 & 13 & 205 & 104 & 104 & 32 & 4 & 18 & 52 & 149 & 67 & 3434 & 5173403 \\
\hline $45-$ & 232 & 471 & 867 & 282 & 1810 & 96 & 1034 & 22 & 254 & 102 & 135 & 59 & 8 & 24 & 71 & 203 & 78 & 5748 & 5115691 \\
\hline $50-$ & 337 & 852 & 1320 & 374 & 2190 & 141 & 1709 & 25 & 339 & 108 & 231 & 80 & 14 & 31 & 131 & 211 & 101 & 8194 & 4047509 \\
\hline $55-$ & 305 & 1102 & 1761 & 430 & 2193 & 166 & 1950 & 30 & 238 & 117 & 166 & 50 & 19 & 41 & 110 & 154 & 126 & 8958 & 2957812 \\
\hline $60-$ & 304 & 1427 & 2166 & 509 & 2120 & 241 & 2523 & 38 & 168 & 94 & 156 & 51 & 26 & 82 & 121 & 169 & 99 & 10294 & 2486122 \\
\hline $65-$ & 359 & 1784 & 2816 & 686 & 2430 & 276 & 3363 & 60 & 184 & 102 & 201 & 62 & 74 & 132 & 150 & 192 & 150 & 13021 & 2125649 \\
\hline $70-$ & 320 & 2046 & 3175 & 876 & 2232 & 328 & 3961 & 69 & 136 & 83 & 153 & 50 & 145 & 185 & 157 & 178 & 147 & 14241 & 1601637 \\
\hline $75-$ & 183 & 1568 & 2526 & 751 & 1692 & 269 & 3045 & 68 & 127 & 64 & 120 & 38 & 140 & 186 & 123 & 126 & 128 & 11154 & 1022733 \\
\hline 80- & 137 & 967 & 1571 & 525 & 1011 & 154 & 1755 & 65 & 72 & 59 & 83 & 25 & 124 & 149 & 80 & 77 & 109 & 6963 & 545697 \\
\hline$>85$ & 72 & 472 & 817 & 332 & 527 & 67 & 930 & 57 & 42 & 35 & 36 & 18 & 104 & 112 & 30 & 39 & 65 & 3755 & 308427 \\
\hline
\end{tabular}

Neoplasms (ICD-10 codes): A: lip, oral cavity, pharynx (C00-C14); B: esophagus (C15); C: stomach (C16); D: colon, rectum (C18-C21); E: liver (C22); F: pancreas (C25); G: trachea, lung (C33-C34); H: skin (C43-C44); I: breast (C50); J: cervix (C53); K: uterus (C54-C55); L: ovary (C56); M: prostate (C61); N: bladder (C67); O: lymphoid, hematopoietic, related tissue (C81-C90, C96); P: leukemia (C91-C95); Q: other malignant neoplasms (C17, C23-C24, C26C32, C37-C41, C45-C49, C51-C52, C57-C60, C62-C66, C68-C80, and C97); R: all cancers.

Table 4 shows the results of the Poisson regression analysis to evaluate the mortality risk of aging with time (social development) for major cancers. We observed variations in the quantitative values of the effect of age with time on diseases. For the 17 cancers, the $B$ value (the interaction between time and age) ranged from -0.169 to 0.435 . A higher value of $B$ indicated a stronger effect on cancer of aging with time (social development). The effect of aging with time was significantly reduced for cancers of the uterus, liver, pancreas, and cervix $(p<0.05)$ and was significantly increased for cancers of the esophagus and leukemia $(p<0.05)$.

According to their positions on the scatter diagram, the 17 neoplasms could be suggested to divide into three groups (Figure 1). Cancers located in the upper right were termed developed cancers (more prevalent in people with a higher quality of life) and comprised three cancers. Cancers in the lower left part were termed undeveloped cancers (more prevalent in people with a lower quality of life) and comprised four cancers. The third group, positioned between the developed and undeveloped groups, was termed as cancers insensitive to social development and comprised ten cancers (58.8\%). Figure 2 illustrates the detailed changes with social development for the three groups of cancers.

The constituent ratios of death from the three groups of cancers are shown in Figure 3. The constituent ratio increased from $4.9 \%$ in 2004 to $7.33 \%$ in 2015 for the developed cancers, decreased from $34.78 \%$ in 2004 to $25.06 \%$ in 2015 for the undeveloped cancers, and was approximately equal (60.84\% in 2004 vs. $67.61 \%$ in 2015$)$ for cancers insensitive to social development. The constituent ratios of death from insensitive to social development cancers were approximately two-thirds of all cancers.

\section{Discussion}

Life expectancy at birth in 2004 was 73.6 years, and in 2015, it was 76.1 years. Therefore, our data for the two time point analysis could reveal the contribution of social development to the development of cancer. The results revealed that these contributions varied without consideration of age. In the 17 cancers studied, the $\operatorname{lnRC}$ value ranged from -1.04 to 0.38 (Table 3), indicating that the contributions of social development to different cancers varied.

The greater the $\operatorname{lnRC}$, the greater the risk of disease risk with time (social development). The $\operatorname{lnRC}$ data showed that among the 17 cancers studied, uterus cancer had the lowest disease risk with time (social development) and cervix cancer had the highest risk. The 17 neoplasms could be divided into developed cancers (for which social development might be a risk factor), undeveloped cancers (for which lack of social development might be a risk factor), and cancers insensitive to social development, according to their position on the scatter diagram (Figure 2). The constituent ratio of death from all cancers increased from $4.9 \%$ in 2004 to $7.33 \%$ in 2015 for developed cancers and decreased from 34.78\% in 2004 to $25.06 \%$ in 2015 for undeveloped cancers (Figure 3). 
TABle 2: Age-stratified number of deaths from major cancers in 2015 in China [22].

\begin{tabular}{|c|c|c|c|c|c|c|c|c|c|c|c|c|c|c|c|c|c|c|c|}
\hline \multirow{2}{*}{ Age } & \multicolumn{18}{|c|}{ Cancers } & \multirow{2}{*}{ Survival } \\
\hline & A & B & $\mathrm{C}$ & $\mathrm{D}$ & E & $\mathrm{F}$ & G & $\mathrm{H}$ & I & J & $\mathrm{K}$ & $\mathrm{L}$ & M & $\mathrm{N}$ & $\mathrm{O}$ & $\mathrm{P}$ & Q & $\mathrm{R}$ & \\
\hline $0-$ & 0 & 0 & 0 & 1 & 14 & 0 & 1 & 0 & 0 & 0 & 0 & 0 & 0 & 0 & 10 & 88 & 15 & 129 & 2804289 \\
\hline $1-$ & 0 & 0 & 0 & 0 & 32 & 0 & 2 & 1 & 0 & 0 & 0 & 0 & 0 & 0 & 42 & 239 & 31 & 347 & 1836631 \\
\hline 5- & 5 & 0 & 1 & 0 & 13 & 2 & 2 & 0 & 0 & 0 & 0 & 0 & 0 & 1 & 39 & 217 & 18 & 298 & 5322497 \\
\hline $10-$ & 4 & 1 & 2 & 2 & 12 & 1 & 5 & 2 & 0 & 0 & 0 & 2 & 0 & 0 & 42 & 207 & 11 & 291 & 12042350 \\
\hline $15-$ & 9 & 1 & 5 & 18 & 33 & 1 & 20 & 5 & 1 & 0 & 2 & 4 & 0 & 1 & 52 & 265 & 14 & 431 & 14609389 \\
\hline $20-$ & 19 & 5 & 33 & 39 & 121 & 10 & 48 & 5 & 9 & 8 & 4 & 8 & 1 & 3 & 57 & 327 & 21 & 718 & 22895079 \\
\hline 25- & 31 & 9 & 125 & 84 & 301 & 21 & 160 & 10 & 70 & 38 & 15 & 16 & 2 & 4 & 128 & 380 & 35 & 1429 & 18670345 \\
\hline $30-$ & 77 & 14 & 209 & 168 & 671 & 43 & 280 & 18 & 165 & 112 & 28 & 34 & 2 & 8 & 99 & 322 & 44 & 2294 & 1678 \\
\hline $35-$ & 152 & 43 & 385 & 292 & 1405 & 72 & 660 & 17 & 335 & 187 & 74 & 53 & 4 & 13 & 143 & 352 & 59 & 4246 & 3806 \\
\hline $40-$ & 338 & 245 & 953 & 623 & 3386 & 241 & 1957 & 62 & 780 & 465 & 128 & 136 & 10 & 34 & 288 & 508 & 119 & 10273 & 67145 \\
\hline $45-$ & 582 & 792 & 1943 & 1104 & 5582 & 479 & 4174 & 78 & 1185 & 836 & 254 & 223 & 15 & 62 & 454 & 624 & 153 & 18540 & 26394480 \\
\hline $50-$ & 737 & 1573 & 2899 & 1470 & 6787 & 759 & 6897 & 79 & 1408 & 938 & 300 & 351 & 44 & 128 & 632 & 670 & 220 & 25892 & 16034245 \\
\hline $55-$ & 804 & 2613 & 4376 & 2035 & 7964 & 1231 & 10417 & 108 & 1343 & 800 & 341 & 331 & 86 & 217 & 774 & 730 & 250 & 34420 & 18285929 \\
\hline $60-$ & 1025 & 4531 & 7151 & 3098 & 9985 & 1703 & 16323 & 153 & 1360 & 892 & 329 & 416 & 211 & 397 & 1109 & 1065 & 337 & 50085 & 14053811 \\
\hline $65-$ & 900 & 5299 & 8031 & 3362 & 8808 & 1832 & 17509 & 192 & 861 & 716 & 339 & 349 & 281 & 520 & 1070 & 896 & 339 & 51304 & 9032555 \\
\hline $70-$ & 733 & 5371 & 8314 & 3536 & 7551 & 1826 & 17546 & 202 & 652 & 594 & 228 & 273 & 508 & 658 & 1041 & 860 & 309 & 50202 & 6728141 \\
\hline $75-$ & 694 & 5160 & 8422 & 4052 & 6600 & 1743 & 17766 & 237 & 587 & 518 & 229 & 211 & 840 & 891 & 932 & 815 & 385 & 50082 & 5350578 \\
\hline $80-$ & 507 & 4122 & 6673 & 3645 & 4893 & 1424 & 13547 & 298 & 469 & 367 & 182 & 147 & 887 & 887 & 645 & 528 & 382 & 39603 & 3320829 \\
\hline$>85$ & 329 & 2607 & 4366 & 2727 & 3064 & 869 & 8259 & 361 & 405 & 250 & 99 & 88 & 826 & 830 & 335 & 270 & 282 & 25967 & 1817600 \\
\hline
\end{tabular}

Neoplasms (ICD-10 codes): A: lip, oral cavity, pharynx (C00-C14); B: esophagus (C15); C: stomach (C16); D: colon, rectum (C18-C21); E: liver (C22); F: pancreas (C25); G: trachea, lung (C33-C34); H: skin (C43-C44); I: breast (C50); J: cervix (C53); K: uterus (C54-C55); L: ovary (C56); M: prostate (C61); N: bladder (C67); O: lymphoid, hematopoietic, related tissue (C81-C90, C96); P: leukemia (C91-C95); Q: other malignant neoplasms (C17, C23-C24, C26C32, C37-C41, C45-C49, C51-C52, C57-C60, C62-C66, C68-C80, and C97); R: all cancers.

TABLE 3: Two time point analysis of the mortality rate for different cancers.

\begin{tabular}{|c|c|c|c|c|c|}
\hline \multirow[t]{2}{*}{ Cancers } & \multicolumn{2}{|c|}{$\begin{array}{c}\text { Standardized mortality rate } \\
\text { (annual deaths per } 10^{5} \\
\text { population) }\end{array}$} & \multirow[t]{2}{*}{$\mathrm{RC}$} & \multirow[t]{2}{*}{$\ln R C$} & \multirow[t]{2}{*}{$p$} \\
\hline & 2004 & 2015 & & & \\
\hline Lip, oral cavity, and pharynx & 2.07 & 1.87 & 0.90 & -0.11 & $<0.001$ \\
\hline Esophagus & 14.02 & 8.21 & 0.59 & -0.53 & $<0.001$ \\
\hline Stomach & 22.89 & 13.80 & 0.60 & -0.51 & $<0.001$ \\
\hline Colon and rectum & 6.62 & 6.68 & 1.01 & 0.01 & $<0.001$ \\
\hline Liver & 24.28 & 18.01 & 0.74 & -0.30 & $<0.001$ \\
\hline Pancreas & 2.37 & 3.14 & 1.32 & 0.28 & $<0.001$ \\
\hline Trachea and lung & 27.41 & 29.59 & 1.08 & 0.08 & $<0.001$ \\
\hline Skin & 0.61 & 0.45 & 0.74 & -0.30 & $<0.001$ \\
\hline Breast & 2.57 & 2.64 & 1.03 & 0.03 & $<0.001$ \\
\hline Cervix & 1.26 & 1.85 & 1.47 & 0.39 & $<0.001$ \\
\hline Uterus & 1.95 & 0.69 & 0.35 & -1.05 & $<0.001$ \\
\hline Ovary & 0.65 & 0.72 & 1.11 & 0.10 & $<0.001$ \\
\hline Prostate & 0.82 & 0.85 & 1.04 & 0.04 & $<0.001$ \\
\hline Bladder & 1.22 & 1.12 & 0.92 & -0.08 & $<0.001$ \\
\hline Lymphoid, hematopoietic tissue & 1.52 & 2.20 & 1.45 & 0.37 & $<0.001$ \\
\hline Leukemia & 3.69 & 3.03 & 0.82 & -0.20 & $<0.001$ \\
\hline Other malignant neoplasm & 1.59 & 0.81 & 0.51 & -0.67 & $<0.001$ \\
\hline All cancers & 123.90 & 106.14 & 0.86 & -0.15 & $<0.001$ \\
\hline
\end{tabular}

$\operatorname{lnRC}$ is the absolute RC (logarithmically transformed RC). A value $<1$ indicated a decreasing trend of mortality with time. 
TABLe 4: The mortality risk from aging with time for major cancers using Poisson regression analysis.

\begin{tabular}{|c|c|c|c|c|}
\hline Group of cancers & & $\mathrm{B}$ & $95 \% \mathrm{CI}$ & $\mathrm{p}$ \\
\hline \multirow[t]{4}{*}{ Undeveloped } & Uterus & -0.144 & $-0.272 \sim-0.017$ & 0.027 \\
\hline & Other malignancy & 0.090 & $-0.046 \sim 0.225$ & 0.196 \\
\hline & Esophagus & 0.164 & $0.112 \sim 0.216$ & $<0.0001$ \\
\hline & Stomach & 0.020 & $-0.019 \sim 0.059$ & 0.322 \\
\hline \multirow[t]{10}{*}{ Insensitive to social development } & Skin & -0.085 & $-0.317 \sim 0.147$ & 0.475 \\
\hline & Liver & -0.052 & $-0.085 \sim-0.019$ & 0.002 \\
\hline & Leukemia & 0.435 & $0.343 \sim 0.528$ & $<0.0001$ \\
\hline & Lip, oral cavity, and pharynx & -0.040 & $-0.130 \sim 0.051$ & 0.390 \\
\hline & Bladder & -0.038 & $-0.245 \sim 0.168$ & 0.717 \\
\hline & Colon and rectum & 0.009 & $-0.058 \sim 0.075$ & 0.798 \\
\hline & Breast & -0.023 & $-0.123 \sim 0.076$ & 0.647 \\
\hline & Prostate & 0.282 & $-0.037 \sim 0.601$ & 0.084 \\
\hline & Lung & -0.028 & $-0.061 \sim 0.005$ & 0.093 \\
\hline & Ovary & -0.041 & $-0.232 \sim 0.149$ & 0.670 \\
\hline \multirow[t]{4}{*}{ Developed } & Pancreas & -0.124 & $-0.233 \sim-0.014$ & 0.027 \\
\hline & Lymphoid, hematopoietic tissue & -0.005 & $-0.130 \sim 0.119$ & 0.931 \\
\hline & Cervix & -0.169 & $-0.304 \sim 0.034$ & 0.014 \\
\hline & All cancers & -0.002 & $-0.017 \sim 0.014$ & 0.809 \\
\hline
\end{tabular}

B: measure of the interaction between time and age (a positive value of B indicates an increased aging risk with time); CI: confidence interval.

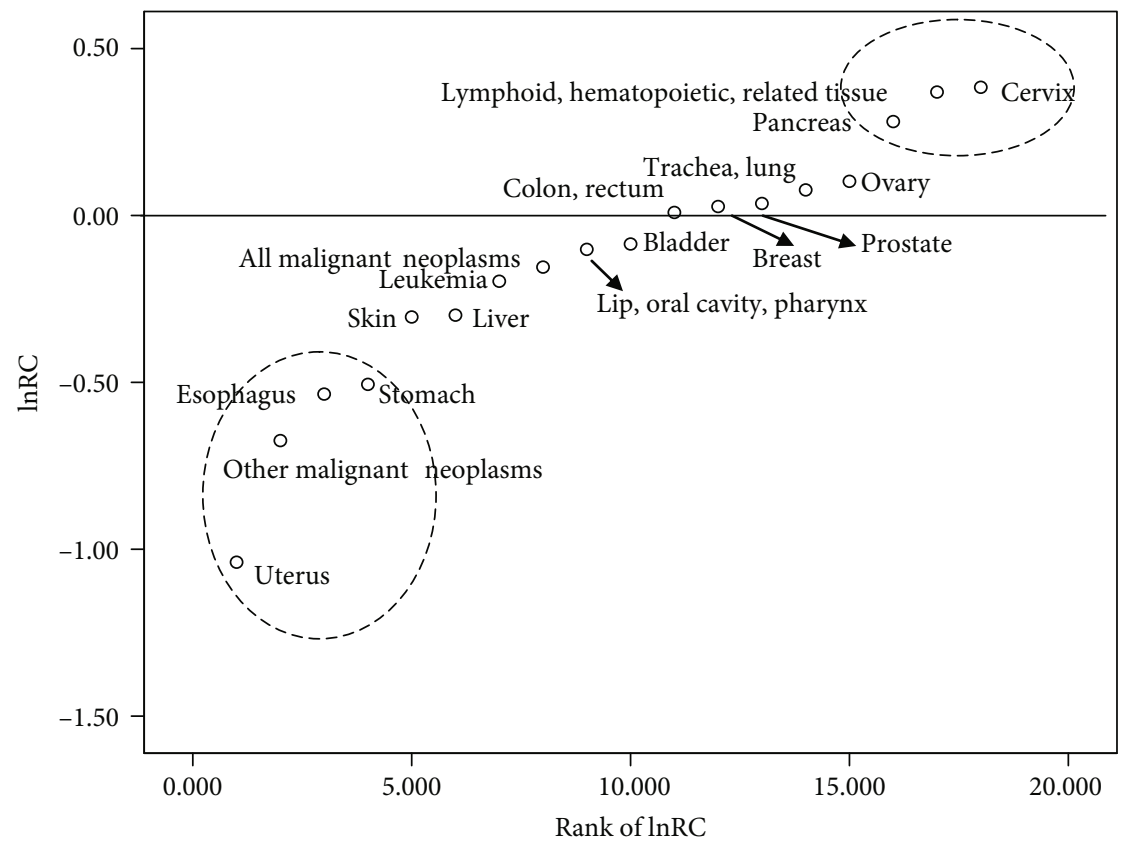

FIGURE 2: Cancer groups classified on the basis of sensitivity to time. RC: ratio of change with time; rank of lnRC: in order of cancers with $\operatorname{lnRC}$ with respect to deaths caused by a particular cancer.

This suggested that the categories of cancer in this study are accurate and reasonable.

Unexpectedly, about $60 \%$ (as assessed by type of cancer) and two-thirds (as assessed by constituent ratio of death from all cancers) of cancers were insensitive to social development, which suggested that the improvement in social development and medical care might have played a limited role in altering the rate of death from cancers. Internal factors, including aging, might have a key effect on cancer occurrence.

Aging is a significant and uncontrollable risk factor for death $[1,24,25]$; therefore, determining the effects of aging on cancers with social development is important. The different contributions of age to mortality from cancers between 2004 and 2015 could also be considered as the change in 
2004

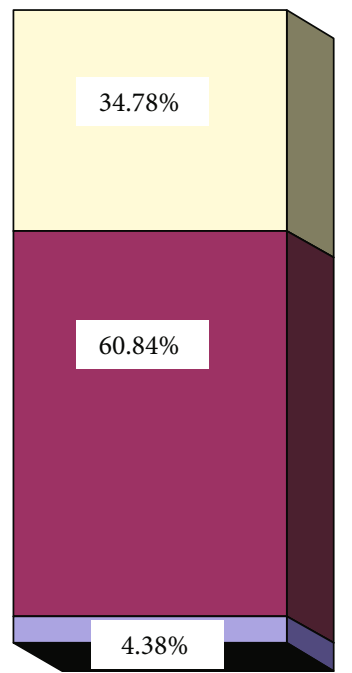

Undeveloped cancers

Developed cancers
2015

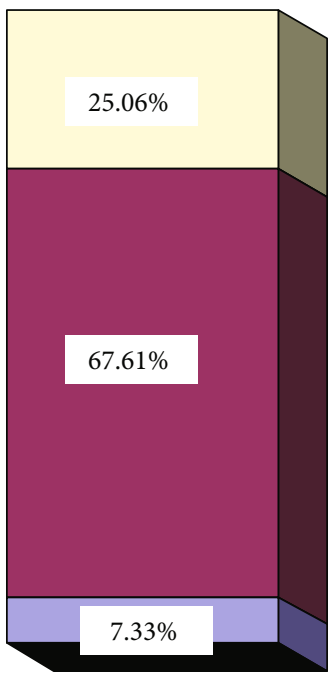

Figure 3: Changes in the constituent ratio with time for the three groups of cancer.

the effect of other risk factors on cancers with time (social development). A larger difference indicated an increased effect of aging or a decreased effect of other risk factors on death resulting from cancers with social development. Therefore, changes in the effect of aging with time implied that the structure of the risk factors had changed with increasing social development. Our results showed that the $B$ value (representing the interaction between time and age) for the 17 cancers ranged from -0.169 to 0.435 . The effect of aging with social development was significantly reduced for cancers of the uterus, liver, pancreas, and cervix $(p<0.05)$ and was significantly increased for cancers of the esophagus and leukemia $(p<0.05)$, suggesting that the structure of risk factors had changed with increasing social development for these cancers. Moreover, for most of the cancers categorized as insensitive to social development, the level of risk and the structure of the risk factors had not changed with time (Figure 3 ).

The identification of a group of developed cancers, including cervix, pancreas, and "lymphoid, hematopoietic, and related tissue" cancers, showed that risk factors from social development have contributed to increases in the rates of some cancers. The risk factors might have a more important role in the relatively young population for cervix and pancreas cancers. Accordingly, an emphasis on avoiding risk factors from social development or urbanization might contribute to disease prevention for these developed cancers.

The presence of a group of undeveloped cancers, including uterus, esophagus, stomach, and other malignant neoplasms, showed that protective factors from social development have contributed to decreases in the rates of some cancers. The protective factors might have a more important role in the relatively young population for esophagus cancer and in the relatively old population for uterus cancer. Accordingly, disease prevention might be aided by an emphasis on public health and increasing basal health status for undeveloped cancers.
One limitation of this study is that the social development was not described with more indicators. Time and social development may not be equal. However, China's gross domestic product was 1.955 trillion USD in 2004 and 11.016 trillion USD in 2015; in 2004, life expectancy at birth was 73.6 years, whereas in 2015 , it was 76.1 years. Therefore, time and social development may be parallel during 2004-2015. Increase of gross domestic product and rapid urbanization would change lifestyle; cancer-related risk would also be changed with this processes. Therefore, time could represent social development in this period. The findings also suggested that only about $30 \%$ of cancers are sensitive to changes in socioenvironmental factors over time. Analyzing and controlling external risk factors might lead to $30 \%$ of cancers being prevented, because the external factors leading to cancers are relatively controllable. Solutions to developed urbanized diseases mainly involve avoiding risk factors, which include tobacco use, overweight, physical inactivity, inadequate diet, sexually transmitted infections, alcohol consumption, and air pollution. General protective interventions for life quality, including nutritional status, lifestyle, and quality of social life, could potentially delay the aging process. Thus, the primary prevention of cancers will be aided by increasing our understanding of the categories of cancer affected by social development. Further studies are warranted to observe correlation between specific risk factors with social development and cancers for developing more available preventative strategies.

\section{Data Availability}

The data used to support the findings of this study are available from the corresponding author upon request.

\section{Conflicts of Interest}

The author declares that he has no conflicts of interest. 


\section{Acknowledgments}

This work was supported by the Special Grant for Scientific and Technological Innovation of Dalian (2018J12SN084).

\section{References}

[1] L. Hui, "Assessment of the role of ageing and non-ageing factors in death from non-communicable diseases based on a cumulative frequency model," Scientific Reports, vol. 7, no. 1, p. 8159, 2017.

[2] L. Gai, H. Liu, J.-h. Cui, W. Yu, and X.-d. Ding, "The allele combinations of three loci based on, liver, stomach cancers, hematencephalon, COPD and normal population: a preliminary study," Gene, vol. 605, pp. 123-130, 2017.

[3] F. Tong, W. Yu, and H. Liu, "Efficient association analysis between colorectal cancer and allelic polymorphisms of HLA-DQB1 by comparison of age of onset," Oncology Letters, vol. 3, no. 3, pp. 517-519, 2011.

[4] W. X. An, Y. X. Fan, X. H. Liang, and H. Liu, "Changes in median ages at death from selected cancer types in relation to HLA-DRB1/DQB1," Asian Pacific Journal of Cancer Prevention, vol. 15, no. 10, pp. 4125-4128, 2014.

[5] L. Hui, "Aging and chronic disease as independent causative factors for death and a programmed onset for chronic disease," Archives of Gerontology and Geriatrics, vol. 60, no. 1, pp. 178$182,2015$.

[6] Y. Zhou, J. Chen, Z. Wang, and H. Liu, "Evaluating the risk of tumors diseases based on measurement of urinary and serumal antioxidants using the new agar diffusion Methods," Oxidative Medicine and Cellular Longevity, vol. 2017, Article ID 6578453, 6 pages, 2017.

[7] H. Liu, "Quantifying the effects of aging and urbanization on major gastrointestinal diseases to guide preventative strategies," BMC Gastroenterology, vol. 18, no. 1, p. 145, 2018.

[8] L. Hui, L. Shijun, Z. Xinyu, W. Yuai, and X. Xiaoting, "Objective assessment of stress levels and health status using routinely measured clinical laboratory parameters as biomarkers," Biomarkers, vol. 16, no. 6, pp. 525-529, 2011.

[9] S. Wenping, L. Ying, L. Song, L. Yuzhong, and L. Hui, "Objective assessment of health or pre-chronic disease state based on a health test index derived from routinely measured clinical laboratory parameters," Journal of Translational Medicine, vol. 13, no. 1, 2015.

[10] X. Zhao, S. Zhu, X. Jia, L. Yu, and H. Liu, "Constructing a waist circumference density index to predict biological age and evaluating the clinical significance of waist circumference density age," Experimental Gerontology, vol. 48, no. 4, pp. 422-426, 2013.

[11] L. Hui, "Chronic diseases and societal development, based on the death-risk index," Epidemiology, vol. 26, no. 1, pp. e9e10, 2015.

[12] G. Xin, L. Shong, and L. Hui, "Effect of genetic and nongenetic factors, including aging, on waist circumference and BMI, and inter-indicator differences in risk assessment," Experimental Gerontology, vol. 60, pp. 83-86, 2014.

[13] A. G. Renehan, A. Flood, K. F. Adams et al., "Body mass index at different adult ages, weight change, and colorectal cancer risk in the National Institutes of Health-AARP cohort," American Journal of Epidemiology, vol. 176, no. 12, pp. 1130-1140, 2012.
[14] R.-M. Feng, Y.-N. Zong, S.-M. Cao, and R.-H. Xu, "Current cancer situation in China: good or bad news from the 2018 Global Cancer Statistics," Cancer Communications, vol. 39, no. 1, p. 22, 2019.

[15] Y. Liang and S. Li, "Landless female peasants living in resettlement residential areas in China have poorer quality of life than males: results from a household study in the Yangtze River Delta region," Health and Quality of Life Outcomes, vol. 12, no. 1, p. 71, 2014.

[16] J. Chen, "Chronic conditions and receipt of treatment among urbanized rural residents in China," BioMed Research International, vol. 2013, Article ID 568959, 8 pages, 2013.

[17] N. Wu, X. Tang, Y. Wu et al., "Cohort profile: the Fangshan Cohort Study of cardiovascular epidemiology in Beijing, China," Journal of Epidemiology, vol. 24, no. 1, pp. 84-93, 2014.

[18] L. Ma, J. Mai, J. Jing et al., "Empirical change in the prevalence of overweight and obesity in adolescents from 2007 to 2011 in Guangzhou, China," European Journal of Pediatrics, vol. 173, no. 6, pp. 787-791, 2014.

[19] Y. Zhang, J. Mo, and C. J. Weschler, "Reducing health risks from indoor exposures in rapidly developing urban China," Environmental Health Perspectives, vol. 121, no. 7, pp. 751$755,2013$.

[20] F. Chan, S. Adamo, P. Coxson et al., "Projected impact of urbanization on cardiovascular disease in China," International Journal of Public Health, vol. 57, no. 5, pp. 849-854, 2012.

[21] N. Hu, Z. Huang, and Y. Jiang, Chinese Center for Disease Control and Prevention. National disease mortality surveillance system, 2004, Military Medical Science Press, 2009.

[22] Y. Wang and Q. Meng, Chinese Center for Disease Control and Prevention. National disease mortality surveillance system, 2015, Science and Technology of China Press, 2016.

[23] World Health Organization, International Statistical Classification of Diseases and Related Health Problems, Tenth Revision, World Health Organization, Geneva, Switzerland, 1992.

[24] T. Cao, N. Xu, Z. Wang, and H. Liu, "Effects of glutathione Stransferase gene polymorphisms and antioxidant capacity per unit albumin on the pathogenesis of chronic obstructive pulmonary disease," Oxidative Medicine and Cellular Longevity, vol. 2017, Article ID 6232397, 8 pages, 2017.

[25] Y. Zhao, S. Li, and H. Liu, "Estimating the survival advantage based on telomere length and serum biomarkers of aging," Journal of Translational Medicine, vol. 15, no. 1, p. 166, 2017. 